\title{
LARGE AREA SPRAYING OF TERAHERTZ MATERIALS ON FREE-STANDING BIOCOMPATIBLE SILK FILMS

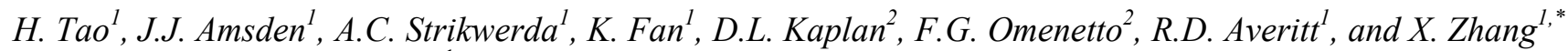 \\ ${ }^{1}$ Boston University, Boston, Massachusetts, USA \\ ${ }^{2}$ Tufts University, Medford, Massachusetts, USA
}

\section{ABSTRACT}

We report the first large area metamaterials patterned on free-standing biocompatible silk substrates using shadow mask evaporation techniques. The as-fabricated silk metamaterial composites show strong electromagnetic resonant responses at terahertz frequencies, providing a promising path to developing a new class of bioelectric and biophotonic devices.

\section{INTRODUCTION}

The strongest natural fiber known, silk is favored by tissue engineers because it is mechanically tough but degrades harmlessly inside the body, offering new opportunities as a robust, bio-compatible and implantable material substrate [1]. To date, most of work has been focused on $2 \mathrm{D}$ or $3 \mathrm{D}$ patterning of the silk films to create biocompatible optical elements such as silk lenses and diffractive gratings [2]. It is desirable to incorporate resonant electromagnetic structures, such as metamaterials, with the silk films. Furthermore, silk fibroin has been proven to be a biologically favorable carrier that enables bio-dopants such as enzymes and proteins to maintain their functionality. This opens the door to a new class of biophotonic devices that could potentially be implanted into the human body to monitor interactions between specific targets and embedded dopants.

Metamaterials are resonant sub-wavelength electromagnetic composites typically consisting of highly conducting metals. Importantly, metamaterials provide the means to design and control both the effective electric permittivity $(\varepsilon)$ and magnetic permeability $(\boldsymbol{\mu})[3]$. The power of metamaterials lies in the fact that it is possible to construct materials with a user designed electromagnetic response (often not available with naturally occurring materials) at a precisely controlled target frequency [4], leading to novel electromagnetic responses which include, but are certainly not limited to, negative refractive index [5], perfect lensing [6], perfect absorbers [7, 8], and invisibility cloaks [9, 10].

This would enable hybrid silk-based sensors that couple bio-functionality with an easily measured electromagnetic response that changes in response to the local environment.

\section{FABRICATION}

The microfabrication on silk films presents unique challenges in comparison to patterning on other widely used substrates such as silicon and PDMS. The main obstacle lies in that potential contamination caused by the conventional photolithography process would dramatically degrade the biocompatibility of the silk films.

\section{Biocompatible Silk Film Preparation}

The typical preparation process of the silk films is shown in Fig. 1. It starts from the processing of silk cocoons, which are cut and cleaned (a), then boiled with sodium carbonate to extract the water soluble sericin (b), dissolved in lithium bromide (c), dialyzed against water to remove the lithium bromide (d) and filtered into a clear water-based silk solution. The silk solution (either undoped or doped with desired biodopants such as enzymes or proteins) is cast on a PDMS mold (e), then cured overnight (f) and detached after transition to the solid phase $(\mathrm{g})$, yielding an optical transparent bio-compatible silk film (h). The thickness could be controlled for various applications by controlling the coating $\&$ curing parameters. In this work, $80 \mu \mathrm{m}$ thick silk films are fabricated.

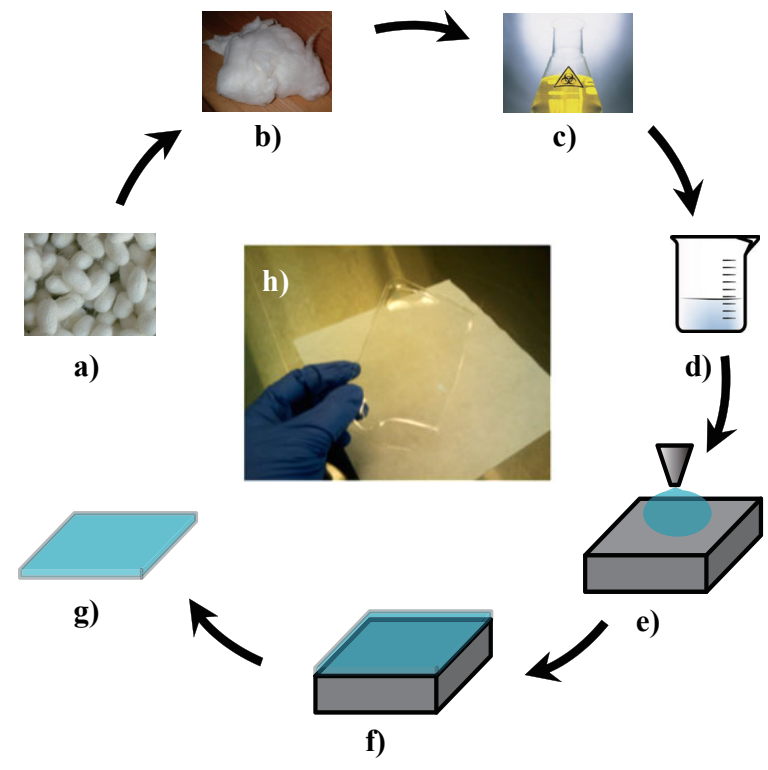

Figure 1: Silk film fabrication process.

Directly Spraying of Metamaterials on Biocompatible Silk Substrates Using Shadow Mask Evaporation Technique

To prevent the contamination of the silk substrate while maintain its bio-compatibility, the silk films are prevented from exposing to any photoresist (PR) and chemical solutions such as acetone and PR developers, which are normally used for typical lift-off metal patterning process.

A shadow mask metal patterning fabrication process was therefore used in this work, as shown in Fig. 2. The shadow mask patterning is based on selective deposition of target material, i.e. gold in our case, through shadow mask, namely micro stencils. The micro stencils with desired metamaterial patterns were fabricated using a 4" silicon wafer with $400 \mathrm{~nm}$ thick LPCVD pre-deposited silicon nitride film on both sides. The split ring resonators (SRRs), widely used as the canonical metamaterial elements, were patterned using lithography and RIE on the top side. Open windows were patterned on the backside and then etched through in $\mathrm{KOH}$ solution to release the structure. Then the micro stencils were attached to the silk films in contact mode, followed by E-beam evaporation of $100 \mathrm{~nm}$ thick gold. The whole evaporation process was controlled under $40^{\circ} \mathrm{C}$, a safe temperature for maintaining the silk quality.

The as-fabricated free-standing samples show good uniformity and mechanical robustness and flexibility, which allow being wrapped into a capsule-like cylinder with a diameter of $\sim 3 \mathrm{~mm}$ with no distortion or cracking observed, as shown in Fig. $3 \& 4$. 


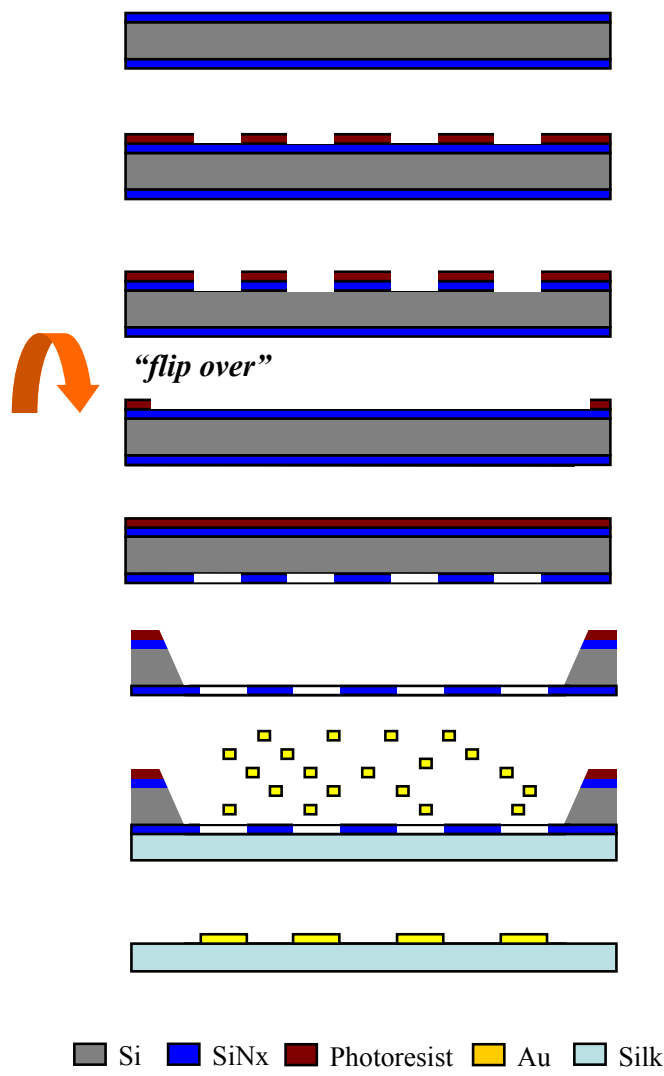

Figure 2: Fabrication process of directly spraying metamaterial structures on silk substrates.

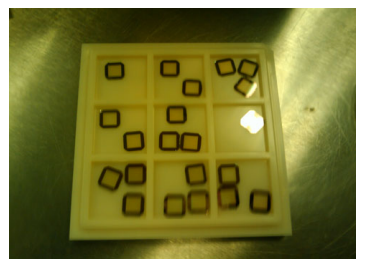

a)

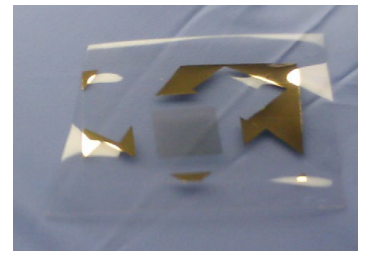

c)

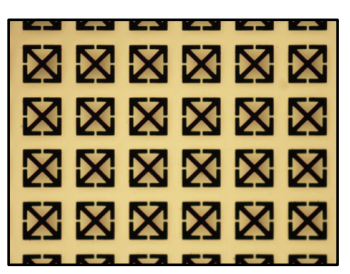

b)

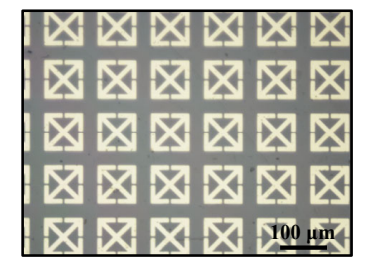

d)
Figure 3: a) Photo of micro metamaterial stencils; b) microscopy photo of one portion of as-fabricated stencil; c) photo of as-fabricated $\mathrm{THz}$ silk metamaterials; d) microscopy photo of one portion of as-fabricated metamaterial array.

The entire fabrication process is conducted in a dry, chemical-free environment preventing any possible contamination that might be involved in other photolithography based metal patterning methods such as lift-off processes and wet etching. Our approach thereby helps to maintain the integrity and biocompatibility of the silk films.

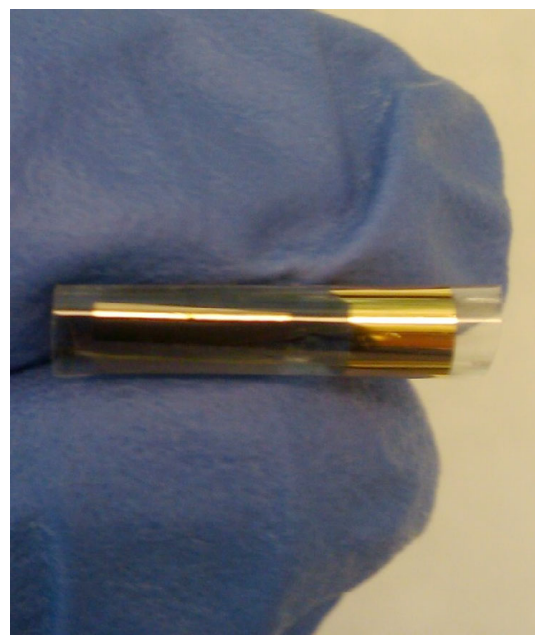

Figure 4: A wrapped "capsule" made of the THz metamaterials patterned on silk films.

\section{CHARACTERIZATION}

Terahertz time-domain spectroscopy (THz-TDS) was used to characterize the electromagnetic response of the metamaterial silk composites. The measurements were performed at room temperature in a dry $(<0.1 \%$ humidity $)$ air atmosphere. The transmission of the $\mathrm{THz}$ electric field was measured for the sample and a reference, which in the present case is simply air. The electric field spectral amplitude and phase were then calculated through Fourier transformation of the time-domain pulses. The spectral transmission was obtained by dividing the sample by the reference, as shown in Fig. 5.

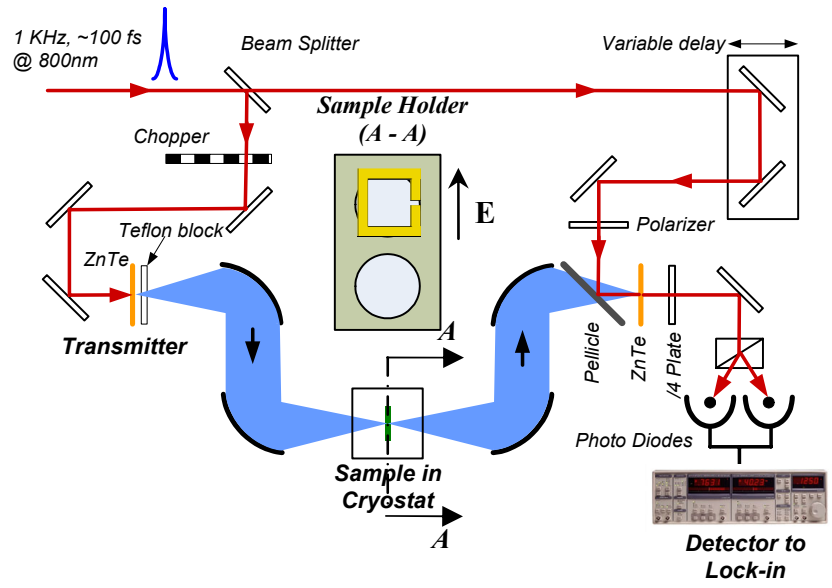

Figure 5: THz Time Domain Spectroscopy (THz-TDS) set up for the metamaterial sample performance characterization. (Top hole: sample; bottom hole: reference/air.)

THz-TDS measurement was first carried out on a series of 80 $\mu \mathrm{m}$ thick pure silk films, which show high field transmission of $\sim$ $60 \%$ of terahertz radiation from $0.15 \mathrm{THz}$ to $1.5 \mathrm{THz}$, as shown in Figure 2a. The refractive index of the silk films $(\mathrm{n}=1.91+\mathrm{i} 0.12$, from $0.15 \mathrm{THz}$ to $1.5 \mathrm{THz}$ ) was then experimentally determined using standard approach described in previous publications, as shown in Fig. 6. 


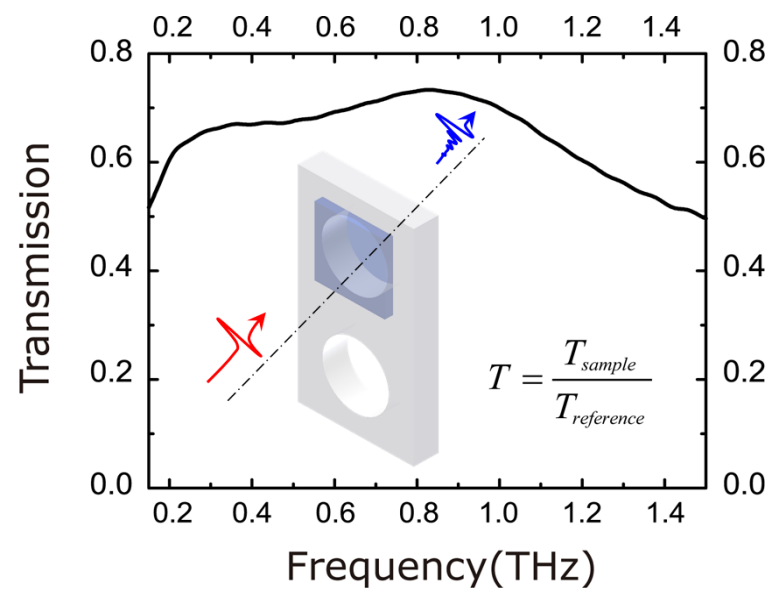

Figure 6: THz-TDS measured transmission spectrum of an 80 micron thick pure silk film without metamaterials.

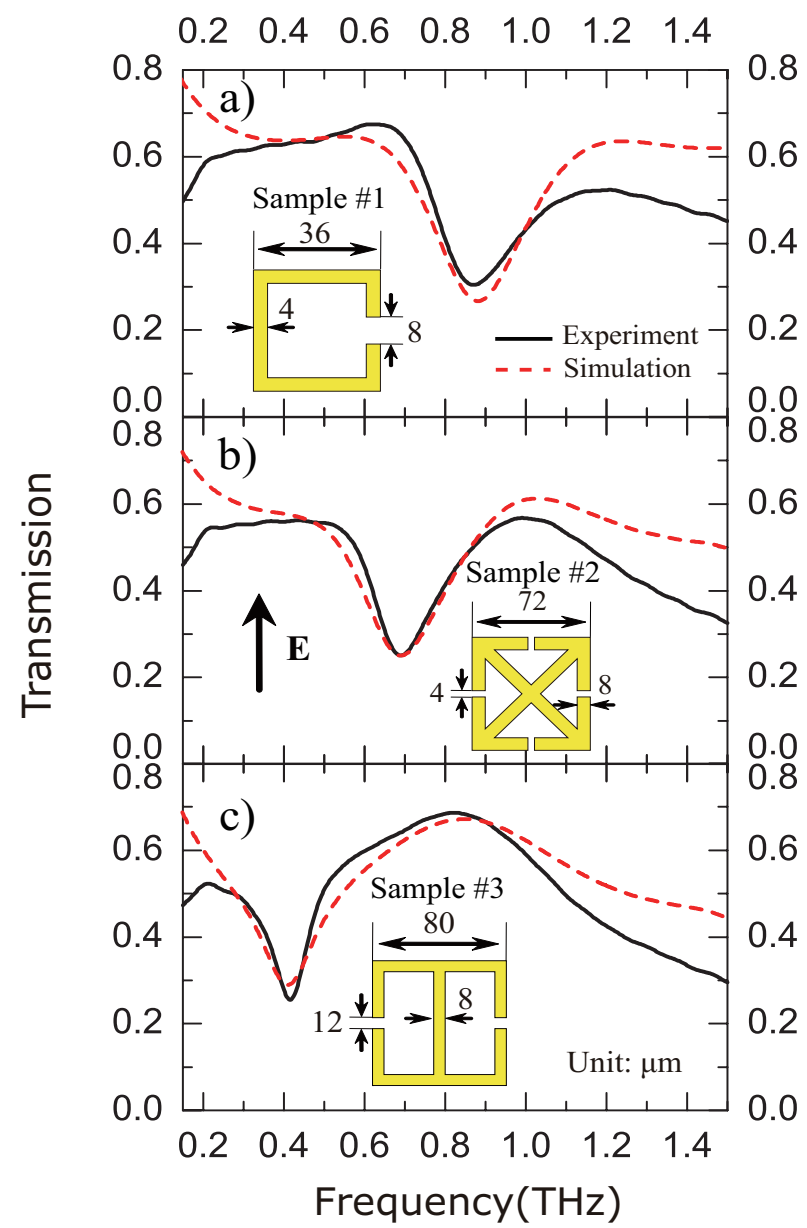

Figure 7: Experiment and simulation results of $\mathrm{THz}$ silk metamaterials, showing strong resonance "fingerprints" in transmission spectrum.

While numerous samples were fabricated and characterized with THz-TDS, we focus on three of samples that include the canonical split ring resonators (\#1, single SRR) and purely electric resonators (\#2, polarization non-sensitive electric resonator and \#3, polarization sensitive electric resonator). Sample \#1 has a unit cell size of $50 \mu \mathrm{m} \times 50 \mu \mathrm{m}$, and samples \#2 \& \#3 have a unit cell of 100 $\mu \mathrm{m} \times 100 \mu \mathrm{m}$. The samples were diced into $1 \mathrm{~cm} \mathrm{x} 1 \mathrm{~cm}$ squares and mounted at normal incidence to the $\mathrm{THz}$ beam with the electric field perpendicular to the SRR gap.

As shown in Fig. 7, sample \#1 has a resonance at $0.85 \mathrm{THz}$, which is, as expected from simulations, higher than the resonance frequencies of sample \#2 at $0.7 \mathrm{THz}$ and sample \#3 at $0.4 \mathrm{THz}$ which have larger unit cells. The experimental results are in reasonable agreement with the simulation data, though noticeable off-resonance disagreement occurs consistently in all three samples, which may arise partly from fabrication imperfections and surface roughness of the samples. All of the samples display strong resonances comparable to those measured on semiconducting and polymer substrates. We note that these resonances are associated with the LC resonant response that arises from circulating currents driven by the electric field that is aligned perpendicular to the SRR gap. That is, the resonant changes in transmission correspond to an effective permittivity.

\section{DISCUSSION}

The SRR, which was used for constituting metamaterials in this work, can be though of as an LC resonator in a simple representation with a resonance frequency of $\omega \sim(1 / \mathrm{LC})^{1 / 2}$, where the inductance (L) results from the current path of the SRR and capacitance $(\mathrm{C})$ is mainly determined by the split gap. Any change in the capacitance or the inductance will result in a change in the resonant response making metamaterials sensitive to the local environment. Therefore, metamaterials would offer an interesting option for integration with silk films for novel sensing and detection applications by providing a natural and potentially biologically active material matrix as the support for the SRR.

The shadow mask evaporation technique as used in this work has stringent requirements on the flatness of the substrates upon which materials are to be deposited. This approach is quite suitable for silk films because the silk substrates exhibit sufficient flatness over large areas resulting from the all-aqueous processing of the protein. This facilitates direct spraying of large area metallic and/or non-metallic patterns with good uniformity onto the silk films. The fabrication process could be readily adopted to fabricate silk metamaterials at other frequencies from the microwave to visible. For example, the technique for making the micro stencils could be easily modified for making nano stencils by switching from standard UV photolithography to electron-beam writing for much smaller features down to tens of nanometers. For isolated patterns with no connecting paths to the stencil supporting frame such as features inside other closed patterns that are difficult to directly deposit in contact mode, it will be possible to use appropriate compensation strategies. The flexibility offered using large area stencil-based deposition in conjunction with available soft fabrication techniques for processing silk films provides a promising path to developing novel flexible electronics on silk substrates.

\section{SUMMARY}

In conclusion, we provide a methodology to fabricate metamaterial structures on silk substrates showing strong resonances at desired frequencies, which opens opportunities for new bio-photonics applications including in vivo bio-tracking, bio-mimicing, implantable biosensor and biodetectors. Furthermore, our results are not limited only to $\mathrm{THz}$ frequencies but may be used over much of the electromagnetic spectrum. Basic technologies are already in place, it's simply a matter of designing the right moulds. 


\section{ACKNOWLEDGEMENTS}

We acknowledge partial support from NSF under Contract No. ECCS 0802036, AFOSR under Contract No. FA9550-09-1-0708, DARPA under Contract No. HR0011-08-1-0044, and DOD/Army Research Laboratory under Contract No. W911NF-06-2-0040. This material is based upon work supported in part by the U.S. Army Research Laboratory and the U.S. Army Research Office under Contract No. W911 NF-07-1-0618 and by the DARPA-DSO. The authors would also like to thank the Photonics Center at Boston University.

\section{REFERENCES}

[1] F.G. Omenetto and D.L. Kaplan, "A New Route for Silk", Nature Photonics, 2, 2008.

[2] J.J. Amsden, P. Domachuk, A. Gopinath, R. White, D.L. Kaplan, L. Dal Negrao, and F.G. Omenetto, "Rapid Nanoimprinting of Silk Fibroin Films for Biophotonics Applications," Advanced Material, 22, 2010.

[3] D.R. Smith, W.J. Padilla, D.C. Vier, S.C. Nemat-Nasser, and S. Schultz, "Composite Medium with Simultaneously Negative Permeability and Permittivity", Physical Review Letters, 84, 2000.

[4] C.M. Bingham, H. Tao, X. Liu, R.D. Averitt, X. Zhang, and W.J. Padilla, "Planar Wallpaper Group Metamaterials for
Novel Terahertz Applications", Optics Express, 16, 2008.

[5] R.A. Shelby, D.R. Smith, S. Schultz, "Experimental Verification of a Negative Index of Refraction", Science, 292, 2001.

[6] J.B. Pendry, "Negative Refraction Makes a Perfect Lens", Physical Review Letters, 85, 2000.

[7] N.I. Landy, S. Sajuyigbe, J.J. Mock, D.R. Smith, and W.J. Padilla, "Perfect Metamaterial Absorber", Physical Review Letters, 100, 2008.

[8] H. Tao N.I. Landy, C.M. Bingham, X. Zhang, R.D. Averitt, and W.J. Padilla, "A Metamaterial Absorber for the Terahertz Regime: Design, Fabrication and Characterization”, Optics Express, 16, 2008

[9] R. Liu, C. Ji, J.J. Mock, J.Y. Chin, T.J. Cui, and D.R. Smith, "Broadband Ground-Plane Cloak", Science, 323, 2009.

[10] D. Schurig, J.J. Mock, B.J. Justice, S.A. Cummer, J.B. Pendry, A.F. Starr, D.R. Smith, "Metamaterial Electromagnetic Cloak at Microwave Frequencies", Science, 314, 2006.

\section{CONTACT}

*X. Zhang, tel: +1-617-358-2702; xinz@bu.edu 